\title{
Polémica por la denominación del Ingeniero Pecuario y de Producción Animal de la Universidad Francisco de Paula Santander
}

\author{
Polemic over the denomination of the Pecuary Engineer and Animal Production of the \\ Francisco de Paula Santander University
}

\section{Controvérsia pela denominação do Engenheiro Pecuário e Engenheiro de Produção Animal da Universidad Francisco de Paula Santander}

\author{
Rubén Darío Carreño-Correa ${ }^{1}$, Julia Carolina Castro-Maldonado ${ }^{2}$
}

Forma de citar: R. D. Carreño, J. C. Castro, "Polémica por la denominación del Ingeniero Pecuario y de Producción Animal de la Universidad Francisco de Paula Santander", Respuestas, vol. 20, no. 1, pp. 6- 15, 2015.

Recibido:

20 de Julio 2014

Aceptado:

13 de Octubre 2014

${ }^{1}$ Magister en Práctica Pedagógica rubendariocc@ufps.edu.co Universidad Francisco de Paula Santander Cúcuta-Colombia

${ }^{2}$ Magister en Gestión de la Calidad de la Educación Superior

Universidad de Pamplona Pamplona-Colombia

\section{Resumen}

Antecedentes: Actualmente las Instituciones de Educación Superior deben actuar con flexibilidad frente a los constantes cambios en los procesos de formación que a su vez se relacionan con la demanda de dar respuesta a las necesidades y problemáticas de la sociedad. Ante esta situación una perspectiva de gran interés para las Universidades, las empresas y los profesionales es el reconocimiento y posicionamiento del egresado. Como estrategia ante la problemática se identifica entre otras alternativas abordar el recurso humano que interviene en el proceso de formación del futuro egresado. Objetivo: Desde esta perspectiva se investigó la percepción que tienen educandos, educadores y egresados respecto a la denominación del programa Ingeniería Pecuaria antes denominada Ingeniería de Producción Animal de la Universidad Francisco de Paula Santander en San José de Cúcuta (Norte de Santander - Colombia). Metodología: Se construyó una historia oral colectiva, enriquecida por un análisis documental. Resultados: Se identificó que Ingeniería Pecuaria surge como una reestructuración de Ingeniería de Producción Animal. Estos dos programas son avalados por el Ministerio de Educación Nacional y el Consejo Profesional Nacional de Ingeniería. A su vez los participantes lo consideran como un programa innovador que busca posicionarse en el sector agropecuario. Además se estableció que el Ingeniero Pecuario y el Ingeniero de Producción Animal al igual que los Zootecnistas son profesionales enfocados a la producción pecuaria. Finalmente se identificó que ninguno de los dos títulos tiene completa aceptación por las entidades generadoras de empleo. Conclusión: Se requiere evidenciar el factor diferenciador del Ingeniero Pecuario con el Zootecnista. Es necesario fortalecer el reconocimiento del Ingeniero de Producción Animal y el Ingeniero Pecuario, situación que amerita analizar las alternativas de conservar la actual titulación, modificarla a ingeniero zootecnista u ofertar el programa como zootecnia.

Palabras clave: Educandos, Educadores, Egresados, Ingeniería de Producción Animal, Ingeniería Pecuaria, Percepción, Zootecnia.

\footnotetext{
Abstract

Background: Currently the institutions of higher education must act with flexibility front to the constants changes in formation processes which in turn are related to the demand
} 
of give answer to the needs and problematic of society. In this situation a perspective of great interest to the universities, the companies and professionals is the recognition and positioning of the graduated. As strategy to the problematic is identified among other alternatives address the human resource that intervenes in formation process of the future graduated. Objective: From this perspective-was investigated the perception that have students, professors and graduates about the tittle of the Pecuary engineering program was investigated. At the beginning, it was named "Animal Production Engineering" from the Francisco de Paula Santander University in San José de Cucuta City (North of Santander - Colombia). Methods: A collective and oral history, enriched by a documentary analysis was made. Results: Was identified that Pecuary Engineering arises as a restructuration Engineering Animal Production. These two programs are supported by the Ministry of National Education and the Council National Professional of engineering. At the same time the participants considers as an innovative program that seeks position itself in the Agropecuary sector. Also was established that the Pecuary Engineer and the Engineer Animal Production just like the Zootechnicians are professionals focused on the Animal Production. Finally was identified that neither of the two titles have complete acceptance for the generating entities of employment. Conclusions: Is show required the differentiator factor with the Zootechnician. Also is relevant manage strategies aimed to strengthening the recognition of engineer of Animal Production and the Pecuary engineer, situation that merits analyze the alternatives of conserve the titling current, modify it to Zootechnician engineer or finally to bid the program as Zootechny.

Keyworks: Learners, Educators, Graduates, Animal Production Engineering, Pecuary Engineering, Perception, Zootechny.

\section{Resumo}

Antecedentes: Atualmente as Instituições de Educação Superior devem atuar com flexibilidade frente às constantes mudanças nos processos de formação, que a sua vez relacionam-se com a demanda de respostas às necessidades e problemáticas da sociedade. Nesta situação uma perspectiva de grande interesse para as Universidades, as empresas e os professionais é o reconhecimento e posicionamento do graduado. Como estratégia nesta problemática se identificam entre outras alternativas, a abordagem do recurso humano que intervém no processo de formação do futuro graduado. Objetivo: Desde esta perspectiva se pesquisou a percepção que tem educandos, educadores e graduados respeito à denominação do programa Engenharia Pecuária antes denominada Engenharia de Produção Animal da Universidad Francisco de Paula Santander em San José de Cúcuta (Norte de Santander - Colômbia). Metodologia: Se construiu uma historia oral coletiva, beneficiada por uma análise documental. Resultados: Se identificou que Engenharia Pecuária surge como uma reestruturação de Engenharia de Produção Animal. Estes dois programas são acreditados pelo Ministério de Educação Nacional e o Conselho Professional Nacional de Engenharia. A sua vez os participantes o consideram como um programa inovador que pretende posicionar-se no sector agropecuário. Além disso, se estabeleceu que o Engenheiro Pecuário e o Engenheiro de Produção Animal ao igual que os Zootecnistas são professionais focalizados à produção pecuária. Finalmente, se identificou que nenhuma das duas titulações tem completa aceitação pelas entidades geradoras de emprego. Conclusão: Se requer evidenciar o fator diferenciador do Engenheiro Pecuário com o Zootecnista. É necessário fortalecer o reconhecimento do Engenheiro de Produção Animal e do Engenheiro Pecuário, situação que deve analisar as alternativas de conservar a atual titulação ou modificá-la para Engenheiro Zootecnista ou ofertar o programa de Zootecnia.

Palavras-chave: alunos, educadores, graduados, Engenharia Pecuária, Engenharia de Produção Animal, percepção, Zootecnia. 
No. 1

Enero - Junio 2015

ISSN 0122-820X

PP: 6-15

8

\section{Introducción}

Acaso la denominación o título profesional importa!; o simplemente el éxito laboral depende de la Universidad que lo otorgue, fortaleciendo así, una posición social en la cual las competencias desarrolladas en el egresado, dejan de ser la base principal para la aceptación del futuro profesional [1]. Aparentemente estos tres criterios son decisivos en la transición del contexto académico al mundo del trabajo; sin embargo, no son los únicos factores incidentes. Indudablemente se pueden plantear múltiples estrategias para dar respuesta a la problemática, una alternativa es abordar educadores, educandos y egresados como recurso humano participante del proceso de enseñanza aprendizaje del profesional, en últimas el sujeto educable puede manifestar pautas valorables de su propio proceso de formación [2].

Fundamentados en la deconstrucción desde el criterio de cuestionar la existencia de posibles falencias en el título otorgado y el proceso de formación desarrollado en el programa Ingeniería Pecuaria (IP) anteriormente denominado Ingeniería de Producción Animal (IPA) [3], el proyecto "Percepción del propósito de formación en Ingeniería de Producción Animal e Ingeniería Pecuaria" desarrollado en la Universidad Francisco de Paula Santander(UFPS) permitió abordar a los docentes, estudiantes y egresados respecto a la problemática existente con la denominación y las diversas reestructuraciones evidenciadas en el programa [4]. Los hallazgos soportan científicamente futuras restructuraciones en los procesos de autoevaluación y el mejoramiento continuo del programa IP de la UFPS.

\section{Materiales y métodos}

Los hallazgos corresponden a un estudio de percepción realizado con estudiantes, docentes y egresados del programa IP e IPA de la UFPS. La investigación fue de tipo descriptivo, con un enfoque cualitativo. Se construyó una historia oral colectiva enriquecida con un análisis documental [5]. Los datos se analizaron con el Software ATLAS.ti 6.0. El muestreo fue de tipo intencional [6], el cual se condicionó a la saturación de los datos, es decir, hasta no percibir nueva información [5].

Se ejecutaron cuatro grupos focales con 48 estudiantes de séptimo a décimo semestre del pensum vigente para IP e IPA. Se aplicó una entrevista semiestructurada abierta en profundidad a siete docentes (incluido un director de plan de estudios) estratificados según la escolaridad y la jerarquía directiva, y a diez egresados (ocho IPA y dos IP de diferentes áreas de conocimiento o desempeño laboral), identificando un profesional por cada dos años de egreso académico.

Documentalmente se analizó el Proyecto Educativo del Programa (PEP) [7], la Licencia Interna de Ingeniería Pecuaria [4] y la Licencia Interna de Ingeniería de Producción Animal [8] vigentes durante el desarrollo de la investigación. Las observaciones se basaron en la grabación de reuniones, conversaciones de "pasillo" y opiniones del recurso humano en espacios diferentes a las entrevistas o grupos focales. La validación se estructuró en la triangulación de fuentes (análisis documental, estudiantes, docentes y egresados); y técnicas e instrumentos (grupos focal, entrevistas, observación) [5].

\section{Resultados y análisis}

El programa IP anteriormente denominado IPA de la UFPS se constituye como un plan de estudios avalado por el Ministerio de Educación Nacional (MEN) con el código SNIES N 51876 vigente hasta el año 2019 [9]. Históricamente para IPA se emitió el código SNIES N 3616 [10].

Las denominaciones de IP e IPA están reglamentadas por la Resolución N³458 de diciembre 30 de 2003 "denominaciones 
compuestas por dos términos, transformación de procesos agropecuarios e Ingeniería, deberán ajustarse a las características... de Ingeniería" [11] y la Resolución $\mathrm{N}^{\circ} 2773$ de noviembre 13 de 2003 al especificar las características de un programa que aplica conocimientos de las ciencias naturales y las matemáticas [12].

El MEN avala a los egresados con el título de Ingeniero Pecuario e Ingeniero de Producción Animal para que puedan y deban expedir la Tarjeta Profesional en el Consejo Profesional Nacional de Ingeniería - COPNIA. Además se identificó que el programa IP se encuentra orientado institucionalmente en constantes procesos de autoevaluación con una proyección hacia la acreditación de alta calidad [7].

Al revisar la reseña histórica de IP contemplada en el PEP, se identificó la existencia de un pensum de IPA (063) vigente para los estudiantes que no solicitaron homologación con la nueva denominación de Ingenieros Pecuarios; y dos pensum de IP, identificados como 163-1 sin vigencia desde el año 2011, y el 163-2 vigente para los estudiantes provenientes de la homologación con el pensum 063 y 163-1, además de los nuevos educandos admitidos en el programa desde el segundo semestre de 2006. De igual forma se verificó que desde el cambio en la denominación del programa se suspendió la oferta de estudiantes nuevos para IPA [7].

Basados enlapercepciónquetieneneducandos, educadores y egresados del programa objeto de la investigación, se estableció que IP es un programa innovador, nuevo, diferente, que busca posicionarse en el sector agropecuario; además se logró establecer que el Ingeniero Pecuario y el Ingeniero de Producción Animal, al igual que el Zootecnista son productores, es decir, profesionales que buscan optimizar las inversiones económicas para maximizar la producción, obteniendo productos con proteína de alta calidad que permitan satisfacer las necesidades del mercado. Según los participantes la diferencia de IP e IPA con Zootecnia debe ser la formación como ingenieros, es decir, la profundidad en asignaturas de formación básica (matemáticas, físicas, químicas) y de ingeniería (software, sistemas de riego, maquinaria, entre otras); además del diseño y reingeniería de construcciones de interés pecuario.

Al respecto se identifica que la percepción del recurso humano participante, coincide parcialmente con el propósito de formación de IPA: "Formar un profesional integral que con la base ingenieril tenga la capacidad de investigar, adaptar y desarrollar las tecnologías propias de los sistemas de producción pecuaria para resolver problemas relacionados con la generación de bienes y servicios de origen animal” [8]; y el objeto de estudio de IP “....son los sistemas de producción animal, entendidos como los procesos de uso y transformación de insumos en productos específicos para el sector pecuario, optimizando la productividad y la rentabilidad, dentro de un contexto de mejoramiento continuo y de conservación de los recursos naturales" [7].

De igual forma se establece similitud con la misión de Zootecnia en la UFPS sede Ocaña, la cual propone formar profesionales capaces de “... resolver con pertinencia y responsabilidad social los problemas del entorno, relacionados con la producción pecuaria... con el propósito de contribuir con el desarrollo nacional e internacional" [13]; el perfil profesional del Zootecnista de la Universidad de Pamplona “...optimizar procesos de producción animal y de transformación y comercialización de productos de origen animal, mediante el uso racional y eficiente de los recursos, con el objetivo de satisfacer las necesidades de la población... mejorando los ingresos de los productores" [14] y el objeto de formación del Zootecnista de la Universidad Nacional
Enero - Junio 2015 ISSN 0122-820X PP: 6-15 
No. 1

Enero - Junio 2015

ISSN 0122-820X

PP: 6-15

10 sede de Bogotá “...formar profesionales que estén en capacidad de promover el desarrollo de las explotaciones pecuarias buscando mejorar los ingresos y el bienestar de los productores y las familias rurales... con la necesidad de conservar el equilibrio natural y la biodiversidad" [15].

En síntesis, IPA, IP y Zootecnia son tres programas académicos que tienden a formar profesionales con un propósito en común: profesionales capaces de optimizar los procesos de transformación de insumos de origen animal en productos pecuarios (productos con proteína de alta calidad) mejorando la rentabilidad del sistema y por ende los ingresos de los productores (optimizar las inversiones para maximizar la producción), contribuyendo así, a dar respuesta con las necesidades de la población relacionadas con la generación de bienes y servicios de origen animal (satisfacer las necesidades del mercado). Sin embargo, los participantes del programa objeto de la investigación no contemplan en específico la conservación de los recursos naturales. Se podría considerar que el aporte al desarrollo de los sistemas pecuarios a nivel regional y nacional es el resultado de optimizar las inversiones para maximizar la producción de alta calidad.

La formación básica y de ingeniería como factor diferenciador propuesto por los participantes es contemplada en el perfil ocupacional del Ingeniero Pecuario "diseña instalaciones para los sistemas de producción animal, buscando la funcionalidad y el bienestar animal" [7] y el propósito de formación del Ingeniero de Producción Animal "formar profesionales que con la base ingenieril..." [8]. Este criterio no se identificó en específico para la formación del Zootecnista en la UFPS sede de Ocaña, la Universidad de Pamplona y la Universidad Nacional.

En relación al desempeño laboral todos los Ingenieros Pecuarios e Ingenieros de
Producción Animal participantes dudaron de las funciones que deben desempeñar en el ejercicio de su carrera, situación que se puede explicar desde la perspectiva planteada en [16], se afirma que "la retórica de la profesionalización, de las competencias, de los procedimientos, construye individuos intercambiables, completamente confundidos con su función, e individuos también constantemente adaptables y re-adaptables, flexibles que se dice ahora". Posiblemente el programa está formando un trabajador flexible [3] que por diversos factores no ha logrado adaptarse a las nuevas tendencias laborales.

En general, estos hallazgos generan controversia con lo reportado en [17], allí se expresa que las instituciones de educación superior han experimentado cambios en los procesos de formación, demandando mayor flexibilidad para adaptarse a las nuevas tendencias de la sociedad y las necesidades que esta presenta, además dentro de los criterios de formación profesional planteados para América Latina en el Proyecto Tuning, se contempla que las instituciones educativas deben interactuar desde la autonomía y la diversidad cultural y académica de cada contexto educativo [18].

Referente al título otorgado al egresado, en todas las intervenciones de los participantes resaltó el orgullo de ser Ingeniero de Producción Animal, argumentándose que a tan solo cinco minutos de Cúcuta, en el país fronterizo Venezuela, existen colegas; además según los participantes, ser Ingeniero de Producción Animal es para gente con gran capacidad y potencial; sin embargo, esta situación ha perdido furor por la tendencia a incrementarse la cantidad de egresados con la nueva denominación de IP.

Desde estaperspectiva y apoyados en el análisis al documento "De profesional a taxista" [19] se puede establecer que el reconocimiento y posicionamiento del programa lo establecen los egresados, especificándose que se requiere 
tiempo y compromiso del profesional, paralelo al apoyo y la retroalimentación institucional. En últimas, como lo afirman los participantes, se necesitan dos o tres cohortes para que a través de un proceso lento la Universidad y el programa logre volver a posicionar a los egresados con la nueva y vigente titulación de IP.

Desde otra perspectiva el título otorgado al egresado generó polémica, ya que para algunos participantes las falencias del programa radican en que IPA fue creado por moda, para igualar las condiciones del momento en el país de Venezuela. De igual forma para otros estudiantes y egresados, es cuestionable el por qué cambiaron la denominación, si IPA aún existe en otros países (Universidad Rafael Urdaneta en Venezuela; Universidad Católica Santa María La Antigua en Panamá; Universidad Autónoma de Santo Domingo en República Dominicana con Ingeniería Agronómica mención Producción Animal); además según los participantes la actual titulación de IP "es poco reconocida por la sociedad y tiende a confundirse con Agropecuaria". En últimas, según los estudiantes participantes no hubo una verdadera reestructuración en la formación del Ingeniero Pecuario respecto al Ingeniero de Producción Animal. Este proceso solo fue un cambio de nombre, en el que resalta negativamente "una menor intensidad horaria presencial para IP".

En general los participantes consensan que "ninguna de las dos denominaciones es realmente reconocida por la sociedad" ya que las entidades gubernamentales y privadas generadoras de empleo o asociaciones como CONVESCOL, por lo general solo reconocen al Zootecnista, Médico Veterinario (MV) o Médico Veterinario Zootecnista (MVZ), generándose convocatorias u ofertas laborales en las cuales no se perfilan Ingenieros Pecuarios o Ingenieros de Producción Animal. Posiblemente como lo manifiestan los participantes "el programa IP aún no es reconocido por falta de gestión del programa y de la Universidad en general".

En la actualidad, posterior a los cambios ya realizados en el programa, para la mayoría de participantes lo más importante no es el nombre de la carrera, ya que ellos afirman que la denominación no es la que hace al profesional o la que genera trabajo, contrario a ello, lo constituye el interés por venderse en el contexto laboral, junto al conocimiento y las capacidades (competencias) que tenga ese nuevo egresado, las cuales dependen de la forma como se halla desarrollado el proceso de enseñanza-aprendizaje que indiscutiblemente debe estar orientado a las necesidades y problemáticas regionales y nacionales, para poder reflejar así, una formación útil para la sociedad. Esta percepción es similar al caso de un profesional Peruano, abogado, "creo que no interesa la universidad dónde se estudia. La profesión la hace cada persona. Pero ahora hay muchos abogados y la gente ya no va a buscarlos" [19].

Al respecto, se identifica que en Colombia el sector de la agricultura, ganadería, caza, silvicultura y pesca ocupa el tercer lugar en generación de trabajo, congregando el 16,8\% del total de personas ocupadas laboralmente a nivel nacional; sin embargo, es una de las actividades con menor crecimiento en términos de generar empleo [20]; paralelo a la disminución del interés de la población en general por estudiar programas de Agronomía, Veterinaria y Afines [21].

Posiblemente para el caso del Ingeniero Pecuario y el Ingeniero de Producción Animal ya existen muchos Zootecnistas, MV y MVZ graduados y poca oferta laboral en el sector agropecuario, además los participantes afirman que "los productores prefieren preguntarse entre ellos, no reconocen económicamente el ejercicio profesional de la asesoría técnica o simplemente Norte de Santander cree más en
Enero - Junio 2015 ISSN 0122-820X PP: 6-15 
No. 1

Enero - Junio 2015

ISSN 0122-820X

PP: 6-15

el profesional que viene de otras regiones del país".

Ante la situación descrita, una alternativa expuesta por los participantes es llegar a Zootecnia, como la mayoría de estudiantes y egresados ha querido y luchado desde el origen del programa. Sin embargo, se identificaron otras opciones como la homologación o doble titulación con Zootecnia, reestructurar el programa a Ingeniería Zootécnica, o realizar una reestructuración profunda de la esencia de formación para mantener y fortalecer el reconocimiento del Ingeniero Pecuario.

Ahora, al retomar que IP es "un programa innovador y exótico, creado para dessaturar el mercado laboral", paralelo a las características de velocidad y cambio que experimenta la actual sociedad del conocimiento, donde la investigación y sus resultados tienden a transformar el núcleo básico de cualquier profesión, surgiendo titulaciones y desapareciendo antiguas ocupaciones a raíz de la incertidumbre del aparente devenir y progreso tecnológico [22], es posible afirmar holísticamente que la solución a la problemática del Ingeniero Pecuario no es un simple cambio de nombre.

De igual forma es pertinente resaltar que de acuerdo a los participantes "actualmente no existe un pensum lo suficientemente sólido para catalogarlo como definitivo, existiendo muchas falencias por corregir", situación en la cual, cambiar una vez más tan solo la denominación no es la solución, ya que es de mayor pertinencia reestructurar criterios de formación (micro-currículos), específicamente la formación de ingeniería. Además se debe tener en cuenta que algunos participantes manifestaron "la UFPS ya ofrece Zootecnia en la sede de Ocaña, razón por la cual, no es lógico que el programa se incline por realizar un nuevo cambio hacia la titulación de Zootecnistas".
Al respecto, en [23] se plantea que un programa amplio y flexible podría preparar al estudiante en papeles de liderazgo y especialidad, en donde la formación en ingeniería podría contar con la suficiente flexibilidad para abarcar distintas aspiraciones profesionales con agilidad permitiendo una rápida transformación, en respuesta a las exigencias sociales del campo de la ingeniería.

Además, todas las profesiones establecen estándares de actuación que pueden ser permanentes o cambiar con el tiempo y el espacio, logrando así, la aceptación en la sociedad o la ausencia de poder de convencimiento desde el actuar del profesional [24]. De esta forma se reitera una vez más la necesidad de reestructurar la esencia de formación del Ingeniero Pecuario y analizar críticamente la posibilidad de mantener la actual denominación o realizar un cambio en la misma.

Finalmente la estrategia de mantener la actual denominación del programa, se fortalece desde la posición de algunos participantes al manifestar que "a los directivos del programa les quedó grande la gerencia de IPA y por eso la reestructuraron a IP, la cual actualmente parece que también les quedó grande, y lo más sencillo es fusilar el programa a Zootecnia o Medicina Veterinaria".

\section{Agradecimientos}

Se agradece a los estudiantes, profesores y egresados de los programas de Ingeniería Pecuaria e Ingeniería de Producción Animal de la UFPS, que participaron en las entrevistas y grupos focales, permitiendo así, la recolección de datos y la re-construcción de la historia.

\section{Conclusiones}

Aunque IP es muy similar a Zootecnia, desafortunadamente no ha tenido el reconocimiento profesional esperado. 
Posiblemente hubiese sido pertinente mantener el programa con la antigua denominación de IPA, sin embargo, en la actualidad no se contempla como una opción.

Es necesario realizar una profunda reestructuración en los lineamientos de formación del Ingeniero Pecuario, fortaleciendo un factor diferenciador con el Zootecnista. Las directivas deben analizar críticamente la posibilidad de conservar el título de Ingeniero Pecuario, cambiarlo a Ingeniero Zootecnista o radicalmente ofertar el programa con la denominación de Zootecnia; en últimas deben gestionar estrategias que contribuyan a solucionar la problemática de reconocimiento del egresado.

\section{Referencias}

[1] I. C. Puentes, "No basta obtener un título profesional", en Tercer foro: Alcance de una reforma de cara a las necesidades del país, ponencia llevada a cabo en el debate de la reforma de la educación superior, Bogotá- Colombia, Mar. 2012.

[2] R. Ávila, "La tarea hermenéutica de las ciencias humanas", Signo y pensamiento, vol. 31, no. 60, pp. 44-60, Sept. 2011.

[3] M. R. Mejía. Educación(es) en la(s) Globalización(es): Entre el pensamiento único y la nueva crítica. Planetapaz, pp. 1-139, 2005.

[4] Comité curricular, programa Ingeniería Pecuaria. "Licencia interna del programa Ingeniería Pecuaria", Universidad Francisco de Paula Santander en Cúcuta, Norte de Santander, Colombia, Oct. 2011.

[5] S. Hernández, C. Fernández,y P. Baptista. Metodología de la investigación. Mac Graw-Hill, pp. 1-182, 2006.

[6] M. Namakforoosh. Metodología de la Investigación. 2a ed. Limusa, p. 189,
2005.

[7] Comité curricular, programa Ingeniería Pecuaria. "Proyecto Educativo del Programa Ingeniería Pecuaria", Universidad Francisco de Paula Santander en Cúcuta, Norte de Santander, Colombia, pp. 1-166, Ago. 2011.

[8] Comité curricular, programa Ingeniería
de Producción Animal. "Licencia interna
del programa Ingeniería de Producción
Animal", Universidad Francisco de
Paula Santander en Cúcuta, Norte de
Santander, Colombia, pp. 1-189, 2007.

Comité curricular, programa Ingeniería
de Producción Animal. "Licencia interna
del programa Ingeniería de Producción
Animal", Universidad Francisco de
Paula Santander en Cúcuta, Norte de
Santander, Colombia, pp. 1-189, 2007.

Comité curricular, programa Ingeniería
de Producción Animal. "Licencia interna
del programa Ingeniería de Producción
Animal", Universidad Francisco de
Paula Santander en Cúcuta, Norte de
Santander, Colombia, pp. 1-189, 2007.

Comité curricular, programa Ingeniería
de Producción Animal. "Licencia interna
del programa Ingeniería de Producción
Animal", Universidad Francisco de
Paula Santander en Cúcuta, Norte de
Santander, Colombia, pp. 1-189, 2007.

Comité curricular, programa Ingeniería
de Producción Animal. "Licencia interna
del programa Ingeniería de Producción
Animal", Universidad Francisco de
Paula Santander en Cúcuta, Norte de
Santander, Colombia, pp. 1-189, 2007.

Comité curricular, programa Ingeniería
de Producción Animal. "Licencia interna
del programa Ingeniería de Producción
Animal", Universidad Francisco de
Paula Santander en Cúcuta, Norte de
Santander, Colombia, pp. 1-189, 2007.

[9] Ministerio de Educación Nacional MEN. Sistema Nacional de Información de la Educación Superior SNIES. Modulo consultas: Programa Ingeniería Pecuaria. 2014. [Online]. Disponible en: http://www.mineducacion.gov. co/sistemasdeinformacion/1735/w3article-218261.html.

[10] Ministerio de Educación Nacional - MEN. Sistema Nacional de Información de la Educación Superior SNIES. Modulo consultas: Programa Ingeniería de Producción Animal. 2014. [Online]. Disponible en: http://snies.mineducacion.gov.co/ consultasnies/ programa/buscar.jsp?con trol $=0.41052432000212347$.

[11] Ministerio de Educación Nacional - MEN. Resolución $\mathrm{N}^{\circ} 3458$ de Diciembre 30 de 2003: Por la cual se definen las características específicas de calidad para la oferta y desarrollo de los programas de formación profesional en Agronomía, Veterinaria y Afines. p. 2, 2003. [Online]. Disponible en: http://www.mineducacion.gov.co/1621/ articles-86406_Archivo_pdf.pdf.

nero - Junio 2015 SN 0122-820X PP: 6-15

: $6-15$ 
No. 1 noviembre 13 de 2003: Por la cual se definen las características específicas de calidad para los programas de formación profesional de pregrado en Ingeniería. 2003. [Online]. Disponible en: http:// www.mineducacion.gov.co/1621/ articles-86417_Archivo_pdf.pdf.

[13] Universidad Francisco de Paula Santander sede Ocaña. Misión del programa de Zootecnia. 2014. [Online]. Disponible en: http://www.ufpso.edu.co/ ofertaufpso/zootecnia.

[14] Universidad de Pamplona. Perfil profesionaldelZootecnista.2014.[Online]. Disponible en: http://www.unipamplona. edu.co/unipamplona/portalIG/home_95/ recursos/01 general/23042014/zootenica. jsp.

[15] Universidad Nacional de Colombia sede Bogotá. Objeto de estudio de la carrera de Zootecnia.2014. [Online]. Disponible en: http://medicinaveterinariaydezootecnia. bogota. unal.edu.co/pregrado/zootecnia. html.

[16] J. Larrosa, “Aprender de oído. Sobre la abolición del sujeto en la Universidad que viene", en Debate liquidación por derribo: leer, escribir y pensar en la Universidad, organizado por La Central. Barcelona- España, abril. p. 16, 2008.

[17] J. Salinas, "Innovación docente y uso de las TIC en la enseñanza universitaria", Revista Universidad y Sociedad del Conocimiento, vol. 1, no. 1, Nov 2004.

[18] P. Beneitone, C. Esquetini, J. Gonzáles, M. Maletá, G. Siufi, R. Wagenaar, "Proyecto Tuning América Latina: Reflexiones y perspectivas de la Educación Superior en América Latina, periodo 2004-2007," Universidad de Deusto y Universidad de Groningen,
Abril 2007. [Online]. Disponible en: http://goo.gl/KL2GQx.

[19] J. Rodríguez, "De profesional a taxista: el mercado laboral de técnicos y profesionales en los 90" ADEC-ATC Asociación Laboral para el Desarrollo, p. 10, 1995. [Online]. Disponible en: http://biblioteca.clacso.edu.ar/clacso/ otros/20111211054326/ rodriguezcuba. pdf.

[20] Departamento Administrativo Nacional de Estadística DANE, "Boletín técnico: Principales indicadores del mercado laboral," Dirección de Difusión, Mercadeo y Cultura Estadística DANE, p. 2, 20, Sept 2014. [Online]. Disponible en: https://www.dane.gov. co/files/investigaciones/boletines/ech/ ech/bol_empleo_ago_14.pdf.

[21] Ministerio de Educación Nacional MEN. Perfil académico y condiciones de empleabilidad: Graduados de educación superior $(2001$ - 2012) y certificados de educación para el trabajo y el desarrollo humano (2010-2012). Observatorio laboral para la educación. 2013. [Online]. Disponible en: http://www. graduadoscolombia.edu.co/html/1732/ articles-195072_perfil_2013.pdf.

[22] A. Villalobos, Y. Melo, “La formación del profesor universitario: Aportes para su discusión," Revista Universidades, vol. 39, pp. 3-20, Oct-Dic 2008.

[23] V. Wani, D. khanduja, "Perspectivas y posibilidades de formación de los ingenieros en India: el fomento de la iniciativa empresarial," Revista Economía Industrial, vol. 362, pp. 135-142, 2006, [Online]. Disponible en: http://www.minetur. gob.es/Publicaciones/ Publicacionesperiodicas/EconomiaIndustrial/RevistaEconomiaIndustrial/362/135.pdf. 
[24] H. Gardner, M. Csikszentmihalyi, W. Damon. Buen trabajo: Cuando ética y excelencia convergen. Barcelona: Ediciones Paidós Ibérica. 2002. 